\title{
Absorption and metabolism of isothiocyanates formed from broccoli glucosinolates: effects of BMI and daily consumption in a randomised clinical trial
}

\author{
Craig S. Charron ${ }^{1 *}$, Bryan T. Vinyard ${ }^{2}$, Sharon A. Ross ${ }^{3}$, Harold E. Seifried ${ }^{3}$, Elizabeth H. Jeffery ${ }^{4}$ and \\ Janet A. Novotny ${ }^{1}$ \\ ${ }^{1}$ US Department of Agriculture, Agricultural Research Service, Beltsville Human Nutrition Research Center, Beltsville, \\ MD 20705, USA \\ ${ }^{2}$ US Department of Agriculture, Agricultural Research Service, Statistics Group, Beltsville, MD 20705, USA \\ ${ }^{3}$ Division of Cancer Protection, National Institutes of Health, National Cancer Institute, Rockville, MD 20852, USA \\ ${ }^{4}$ Department of Food Science and Human Nutrition, University of Illinois, Urbana, IL 61801, USA
}

(Submitted 22 March 2018 - Final revision received 10 September 2018 - Accepted 12 September 2018)

\section{Abstract}

Sulphoraphane originates from glucoraphanin in broccoli and is associated with anti-cancer effects. A preclinical study suggested that daily consumption of broccoli may increase the production of sulphoraphane and sulphoraphane metabolites available for absorption. The objective of this study was to determine whether daily broccoli consumption alters the absorption and metabolism of isothiocyanates derived from broccoli glucosinolates. We conducted a randomised cross-over human study ( $n$ 18) balanced for BMI and glutathione $S$-transferase $\mu 1$ (GSTM1) genotype in which subjects consumed a control diet with no broccoli (NB) for $16 \mathrm{~d}$ or the same diet with $200 \mathrm{~g}$ of cooked broccoli and $20 \mathrm{~g}$ of raw daikon radish daily for $15 \mathrm{~d}$ (daily broccoli, DB) and $100 \mathrm{~g}$ of broccoli and $10 \mathrm{~g}$ of daikon radish on day 16. On day 17 , all subjects consumed a meal of $200 \mathrm{~g}$ of broccoli and $20 \mathrm{~g}$ of daikon radish. Plasma and urine were collected for $24 \mathrm{~h}$ and analysed for sulphoraphane and metabolites of sulphoraphane and erucin by triple quadrupole tandem MS. For subjects with BMI $>26 \mathrm{~kg} / \mathrm{m}^{2}$ (median), plasma AUC and urinary excretion rates of total metabolites were higher on the NB diet than on the DB diet, whereas for subjects with BMI $<26 \mathrm{~kg} / \mathrm{m}^{2}$, plasma AUC and urinary excretion rates were higher on the DB diet than on the NB diet. Daily consumption of broccoli interacted with BMI but not GSTM1 genotype to affect plasma concentrations and urinary excretion of glucosinolate-derived compounds believed to confer protection against cancer. This trial was registered as NCT02346812.

\section{Key words: Broccoli: Glucosinolates: Isothiocyanates: Erucin: Sulphoraphane}

Consumption of Brassica vegetables has been inversely associated with the incidence of several cancers, including cancer of the breast, colon, endometrium, liver, lung, ovaries and stomach $^{(1-8)}$. Brassica vegetables contain numerous healthpromoting compounds, but the ability to disrupt cancer initiation and progression is thought to originate with a class of sulphur-containing secondary metabolites called glucosinolates, which are found in plants of the order Brassicales and in all Brassica vegetables. Glucosinolates have little or no bioactivity, but in the presence of plant or bacterial myrosinase they are hydrolysed to bioactive isothiocyanates and further metabolites.

Isothiocyanates are electrophilic compounds that may inhibit cancer by a variety of mechanisms. Several plant-derived isothiocyanates activate nuclear factor (erythroid-derived 2)-like 2
(NRF2) signalling to induce enzymes involved in maintaining intracellular redox capacity and phase II detoxification enzymes such as $\mathrm{NAD}(\mathrm{P}) \mathrm{H}$ quinone oxidoreductase 1 (NQO1) and glutathione $S$-transferases $(\mathrm{GST})^{(\mathrm{O}, 10)}$. Sulphoraphane (SF, 1-isothiocyanato-4methylsulfinyl butane), an isothiocyanate derivative of glucoraphanin, which is the predominant glucosinolate in broccoli, induces apoptosis $^{(11)}$ and preferentially inhibits histone deacetylase in cancer cells ${ }^{(12)}$. Another chemopreventive mechanism mediated by isothiocyanates may involve NRF2-independent modulation of inflammasomes, which have been implicated in inflammatory disorders and may be linked to some cancers ${ }^{(13)}$.

The effectiveness of isothiocyanates to inhibit cancer may depend on isothiocyanate concentration, molecular mechanism and cell type. Expression of NQO1 doubled in Hepa 1c1c7 cells

Abbreviations: BHNRC, Beltsville Human Nutrition Research Center; DB, daily broccoli; ER, erucin; GSTM1, glutathione S-transferase $\mu$ 1; NB, no broccoli; SF, sulforaphane.

* Corresponding author: C. S. Charron, fax +1 3015049098 , email craig.charron@ars.usda.gov 
at SF concentrations of $0 \cdot 4-0 \cdot 8 \mu \mathrm{m}^{(14)}$, whereas at concentrations of $5-50 \mu \mathrm{M}$, in these cells and HT29 cells, apoptosis was induced dose dependently ${ }^{(15)}$. In a human clinical study, consumption of high-SF broccoli soup, cooked briefly to ensure retention of plant myrosinase activity, up-regulated several genes in gastric mucosa with oxidoreductase activity, whereas broccoli soup with one-third the amount of SF induced only one gene from this group ${ }^{(16)}$.

Therefore, to develop dietary recommendations to optimise the health benefits of Brassica vegetable consumption, it is important to identify factors that influence isothiocyanate absorption and metabolism in humans. There is evidence, for example, that the efficiency of isothiocyanate metabolism is influenced by the presence or absence of the glutathione $S$-transferase $\mu 1$ (GSTM1) and glutathione $S$-transferase $\theta 1$ (GSTT1) genes ${ }^{(17,18)}$. In addition, the content of glucosinolates and other phytochemicals varies considerably across cultivars, impacting effective dose from a serving and therefore affecting bioactivity, as well as blood levels and urinary excretion of isothiocyanates and their metabolites ${ }^{(19)}$. Furthermore, SF is absorbed more rapidly and is found at higher levels in blood and urine following consumption of raw broccoli compared with cooked broccoli, a difference that is likely to be related to the denaturation of plant myrosinase by the heat of cooking $^{(20,21)}$. Some differences in the absorption, metabolism and subsequent urinary excretion of isothiocyanates may be explained by differences in the myrosinase-like activity of gut microbiota among individuals ${ }^{(22)}$. A recent pre-clinical study has shown that adaptation to Brassica vegetables influences absorption and metabolism of isothiocyanates. Microbiota from rats that were given broccoli powder daily for 1 or 2 weeks more efficiently converted glucosinolates to isothiocyanates compared with microbiota from rats with no prior exposure to broccoli powder ${ }^{(23)}$. However, little is known about how dietary patterns affect the absorption and metabolism of isothiocyanates from Brassica vegetables in humans.

Thus, we conducted a human clinical trial to investigate how daily consumption of broccoli might influence absorption and metabolism of isothiocyanates and their metabolites, as reflected in plasma and urinary excretion, and whether any effects were related to BMI, GSTM1 genotype or sex. We targeted SF and its mercapturic acid pathway conjugates because of the large body of evidence supporting the chemopreventive action of SF. We also measured erucin (ER) mercapturic acid pathway conjugates because ER and SF have been shown to interconvert, and thus ER metabolites are related to the pool of SF metabolites $^{(24,25)}$ (Fig. 1).

\section{Methods}

\section{Subjects}

This study was conducted at the Beltsville Human Nutrition Research Center (BHNRC) in Beltsville, MD, USA. Healthy adults were recruited from the Washington, DC area from January to March 2015. Participants were screened to ensure general health and were 37-65 years of age (online Supplementary Fig. S1) with a BMI between 19 and $37 \mathrm{~kg} / \mathrm{m}^{2}$ (online
Supplementary Fig. S2). Potential subjects who met any of the following criteria were excluded: (1) pregnant or lactating; (2) allergic or intolerant to Brassica vegetables; (3) history of bariatric surgery or nutrient malabsorption disease (such as celiac disease) or other metabolic disorders requiring special diet recommendations; (4) used tobacco products; (5) type 2 diabetes requiring the use of diabetes pills, insulin or non-insulin shots; (6) used blood-thinning medications such as Coumadin (warfarin), dicumarol or Miradon (anisindione) and (7) selfreport of alcohol or substance abuse within the past 12 months and/or current acute treatment or rehabilitation program for these problems. Eligibility was determined by routine blood and urine screening and health history questionnaire. Potential subjects were genotyped for GSTM1 and GSTT1. See Fig. 2 for the recruitment and enrollment data. This study was conducted according to the guidelines laid down in the Declaration of Helsinki, and all procedures involving human subjects were approved by the MedStar Health Research Institute (Hyattsville, MD, USA). Written informed consent was obtained from all subjects. Participant characteristics are reported in Table 1. All subjects were GSTT1-present. This trial was registered at clinicaltrials.gov (NCT02346812).

\section{Broccoli}

The broccoli used in this study was procured as a single shipment of commercially frozen broccoli that was thoroughly mixed and stored at $-80^{\circ} \mathrm{C}$. Commercially processed frozen broccoli is blanched and has very little myrosinase activity, particularly when it is further cooked ${ }^{(26)}$. Therefore, broccoli portions consumed during the study were served with raw daikon radish as a source of plant myrosinase. A single batch of fresh raw daikon radish was procured, and slices totalling $10 \mathrm{~g}$ were stored at $-80^{\circ} \mathrm{C}$ and used throughout the study. We chose this approach because it was not possible to obtain fresh broccoli of consistent glucosinolate content over the duration of this study.

Glucosinolates were measured in randomly selected 100-g samples ( $n$ 3) of broccoli by a previously described method before the beginning of the study ${ }^{(27)}$. Broccoli weighing $200 \mathrm{~g}$ provided $97.5 \mu \mathrm{m}$ of glucoraphanin and $5.8 \mu \mathrm{M}$ of glucoerucin (Table 2). Fibre content was determined by the method of Prosky et $a l{ }^{(28)}$.

\section{Experimental design and treatments}

This study was a randomised cross-over design consisting of two 18 -d periods separated by a 3 -week washout. The crossover design was chosen so that each subject could serve as his or her own control, thus increasing the likelihood that differences could be detected. Sample size was estimated for treatment main effects on plasma time curve (AUC) based on data from humans after adaptation to polyphenols in a study conducted in our laboratory. Using the $65 \%$ increase in AUC that we measured ${ }^{(29)}$ and using $80 \%$ power and $P<0.05$, a sample size of 14 was calculated. To allow for drop-out rate, we enrolled eighteen subjects. Power calculations were performed using SigmaPlot 12.0. Subjects consumed either a control diet 
<smiles>CS(=O)CCCCC([AsH2])=[N+]([O-])[O-]</smiles><smiles>CC(CC[N+](=O)[O-])C[SiH](C)C</smiles>

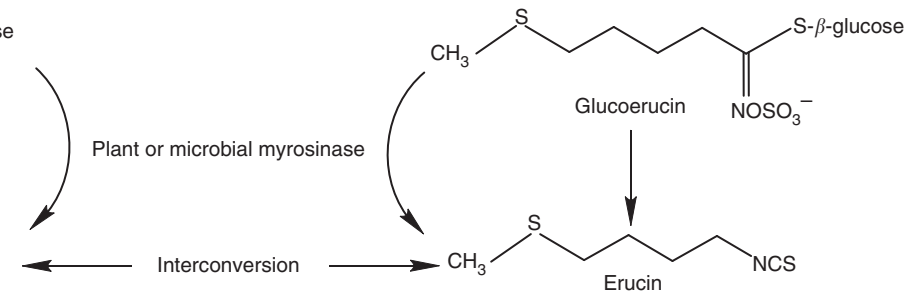<smiles>CS(=O)CCCCNC(=S)[Ge](Cl)(Cl)Cl</smiles><smiles>CS(=O)CCCCNC(=S)OCl</smiles>
Sulforaphane-cysteineglycine<smiles>CCCCCNC(=S)OCCCCC(C)=O</smiles>

Sulforaphane-cysteine<smiles>CC(=O)OC(=S)NCCCCS(C)=O</smiles><smiles>CSCCCCNC(=S)[Ge](Cl)(Cl)Cl</smiles><smiles></smiles><smiles>CCOC(=S)NCCCCSC</smiles><smiles>CCOC(=O)OC(=S)NCCCCSC</smiles>

Fig. 1. Myrosinase-mediated catabolism of glucoraphanin and glucoerucin to sulphoraphane and erucin, respectively, and subsequent mercapturic acid pathway conjugations and interconversion of sulforaphane and erucin.

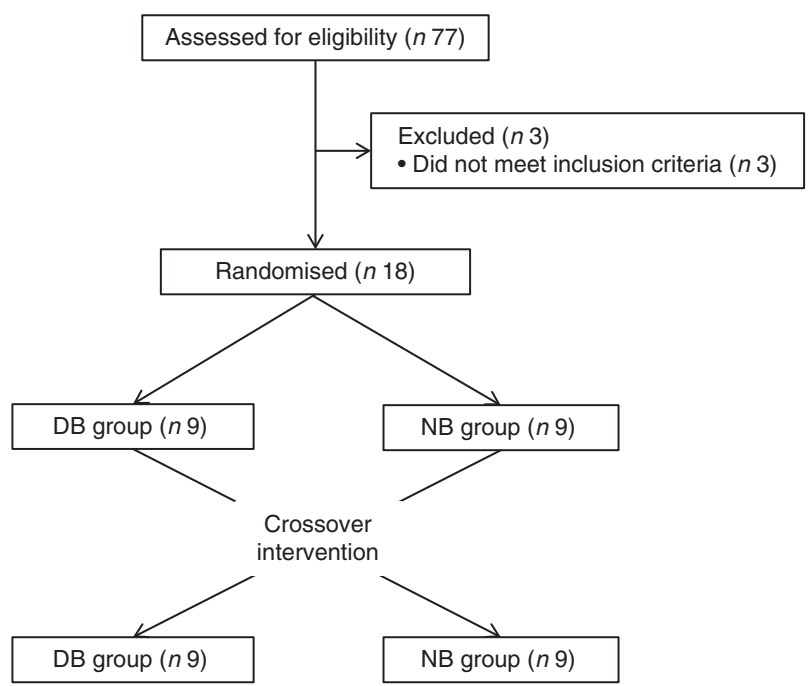

Fig. 2. Recruitment and enrollment of subjects. DB, daily broccoli; NB, no broccoli.

with no broccoli or other Brassica vegetables (NB) or a daily broccoli (DB) diet for $16 \mathrm{~d}$. The NB diet consisted of $16 \%$ energy content from protein, $30 \%$ from fat and the remainder from carbohydrates. Macronutrient content is reported in Table 2. Foods were scaled in $836 \mathrm{~kJ}(200 \mathrm{kcal})$ increments to meet individual energy requirements and to maintain subject weights. The diet consisted of typical American foods and provided 3-5 servings of fruit or vegetables daily $(268-536 \mathrm{~g} / \mathrm{d})$. The DB diet consisted of the same NB diet plus $100 \mathrm{~g}$ of broccoli and $10 \mathrm{~g}$ of frozen raw daikon radish with breakfast and the same amounts of broccoli and daikon radish with dinner each day for $15 \mathrm{~d}$. Broccoli that was consumed at the BHNRC was thawed at $4{ }^{\circ} \mathrm{C}$, and $100-\mathrm{g}$ portions were heated for $30 \mathrm{~s}$ in a $1200-\mathrm{W}$ microwave oven (NE-1258R; Panasonic). For broccoli consumed outside of BHNRC (weekends), subjects were instructed to heat the 100-g broccoli portion in a microwave oven for 15-s increments until warm. On day 16, subjects on the DB diet received $100 \mathrm{~g}$ of broccoli and $10 \mathrm{~g}$ of radish at breakfast but not at dinner. Broccoli was excluded from the day-16 dinner so that metabolites would not carry over to day 17 . On day 17 , all subjects consumed $200 \mathrm{~g}$ of broccoli, $20 \mathrm{~g}$ of daikon radish, a 100-g roll and $10 \mathrm{~g}$ of margarine for breakfast. The broccoli was heated for $30 \mathrm{~s}$ in a $1200-\mathrm{W}$ microwave oven, stirred and heated for an additional 20 s. Each subject received each treatment and was randomly allocated to one of two groups using stratified randomisation to ensure balance of GSTM1 genotype, BMI, sex and age. The allocation sequence was concealed from the principal investigator (C. S. C.) and was assigned diet by the supervisory dietitian without consulting the PI. The allocation sequence was not provided to the PI until the study was completed. Laboratory analysts were blinded to the treatment allocation, although it was not possible to blind the subjects to which diet they received.

Study participants were instructed to consume all foods and only foods provided by the BHNRC, with the exception of 
Table 1. Characteristics of study participants* (Mean values and standard deviations)

\begin{tabular}{|c|c|c|c|c|c|}
\hline \multirow[b]{2}{*}{ GSTM1 genotype } & \multirow[b]{2}{*}{$n$} & \multicolumn{2}{|c|}{ Age (years) } & \multicolumn{2}{|c|}{ BMI $\left(\mathrm{kg} / \mathrm{m}^{2}\right)$} \\
\hline & & Mean & SD & Mean & SD \\
\hline GSTM1-present & 9 & $56 \cdot 6$ & 4.4 & $28 \cdot 1$ & $6 \cdot 2$ \\
\hline Male & 4 & $56 \cdot 3$ & 4.4 & $27 \cdot 8$ & 4.7 \\
\hline Female & 5 & $56 \cdot 8$ & $5 \cdot 2$ & 28.4 & $7 \cdot 7$ \\
\hline GSTM1-null & 9 & $54 \cdot 1$ & $9 \cdot 6$ & $26 \cdot 9$ & 4.0 \\
\hline Male & 4 & $56 \cdot 3$ & $13 \cdot 1$ & $28 \cdot 6$ & 3.4 \\
\hline Female & 5 & $52 \cdot 4$ & 6.9 & 25.5 & $4 \cdot 3$ \\
\hline
\end{tabular}

GSTM1, glutathione S-transferase $\mu 1$

* All subjects were GSTT1-present.

Table 2. Macronutrients, fibre, glucoraphanin and glucoerucin provided by base diet and broccoli

\begin{tabular}{lcc}
\hline & $\begin{array}{c}\text { Base diet } \\
\text { (based on 2000 kcal } \\
(8368 \mathrm{~kJ})\end{array}$ & $\begin{array}{c}\text { Broccoli } \\
\text { (based on 200 g) }\end{array}$ \\
\hline Protein $(\mathrm{g})$ & 82.9 & $6 \cdot 2$ \\
Carbohydrate $(\mathrm{g})$ & 269.0 & $10 \cdot 7$ \\
Fat $(\mathrm{g})$ & 68.5 & 0.2 \\
Fibre $(\mathrm{g})$ & 24.7 & $5 \cdot 0$ \\
Glucoraphanin $(\mu \mathrm{mol})$ & 0 & 97.5 \\
Glucoerucin $(\mu \mathrm{mol})$ & 0 & $5 \cdot 8$ \\
\hline
\end{tabular}

coffee, tea and diet soda. Breakfast and dinner on weekdays were consumed in the BHNRC dining room, and lunches and weekend meals were packed for carryout. Coffee and tea intake was limited to $2 \mathrm{cups} / \mathrm{d}$, and intake of diet soda was not limited. Consumption of coffee, tea and soda was recorded by study participants. Study participants were asked to abstain from Brassica vegetables (except during the DB diet period when they consumed broccoli) and vitamin and mineral supplements beginning 3 weeks before the study and continuing for the duration of the study, including the washout period. No adverse effects were observed. All participants completed the study, and corresponding samples were analysed.

\section{Glutathione S-transferase $\mu 1$ and glutathione S-transferase} $\theta 1$ genotyping

Genotyping was conducted at the Bionomics Research and Technology Center of the Environmental and Occupational Health Sciences Research Institute (Piscataway, NJ) using a previously described method ${ }^{(30)}$.

\section{Sample collection and analysis}

On day 17, blood and urine were collected for $24 \mathrm{~h}$. Blood was collected immediately before the broccoli challenge meal and at $0 \cdot 5,1 \cdot 0,1 \cdot 5,2 \cdot 0,2 \cdot 5,3 \cdot 0,3 \cdot 5,4 \cdot 0,5 \cdot 0,6 \cdot 0$ and $24 \mathrm{~h}$ thereafter. Blood was collected into EDTA-coated vacutainers, centrifuged at $2000 \mathrm{~g}$ for $10 \mathrm{~min}$ and after removing $0 \cdot 5-\mathrm{ml}$ aliquots of plasma into cryovials the cryovials were immediately snapfrozen in liquid $\mathrm{N}_{2}$ and stored at $-80^{\circ} \mathrm{C}$ until analysis. All urine samples were collected and weighed beginning before the broccoli challenge meal and continuing for $24 \mathrm{~h}$. Urine was collected at 2, 4 and $6 \mathrm{~h}$ at BHNRC and aliquots of $1.5 \mathrm{ml}$ were added to $0.25 \mathrm{ml}$ of $0.7 \%$ ascorbic acid and stored at $-80^{\circ} \mathrm{C}$. From 6-14h, subjects collected all urine into a 3-litre collection jug containing $2 \mathrm{~g}$ of ascorbic acid and from 14 to $22 \mathrm{~h}$ collected all urine into a different 3-litre collection jug containing $1 \mathrm{~g}$ of ascorbic acid. A third jug was provided in the event that subjects needed to void before arriving at BHNRC, which was then added to the urine collected at BHNRC at $24 \mathrm{~h}$. Aliquots of $1.5 \mathrm{ml}$ from the 6- to $14-\mathrm{h}$ and $14-$ to $22-\mathrm{h}$ containers were dispensed into cryovials (with no additional acidification), and $1 \cdot 5-\mathrm{ml}$ aliquots from the $24 \mathrm{-h}$ combined collection (included urine from 22 to $24 \mathrm{~h}$ ) were dispensed into cryovials containing $0.25 \mathrm{ml}$ of $0.7 \%$ ascorbic acid. Urine aliquots of $4.0 \mathrm{ml}$ were removed from the 14- to 22 -h collection for the lactulose:mannitol (LM) test. All samples were stored at $-80^{\circ} \mathrm{C}$ until analysis.

\section{Lactulose:mannitol test}

To test for treatment effects on intestinal permeability, subjects were provided and instructed to drink a $100-\mathrm{ml}$ solution containing $5 \mathrm{~g}$ of mannitol and $10 \mathrm{~g}$ of lactulose after their final void at $14 \mathrm{~h}$, which was after fasting for approximately $4 \mathrm{~h}$. Urine from the 14- to 22-h collection was analysed for lactulose and mannitol according to the procedure of Miki et al. ${ }^{(31)}$. Lactulose and mannitol were separated on a Kromasil column $\left(4.6 \times 250 \mathrm{~mm}, 5 \mu \mathrm{m}\right.$; Sigma) at $25^{\circ} \mathrm{C}$ with a mobile phase of $75 \%$ acetonitrile and flow rate of $0.8 \mathrm{ml} / \mathrm{min}$. Standard curves were generated to quantify lactulose and mannitol concentrations.

\section{Analysis of sulforaphane and conjugates of sulforaphane and erucin in plasma and urine}

SF and conjugates of SF and ER were measured in plasma and urine using a previously reported method ${ }^{(32)}$. Aliquots of $0.5 \mathrm{ml}$ of ice-cold plasma were combined with $50 \mu \mathrm{l}$ of $100 \mu \mathrm{M} N$-acetyl ( $N$-butylthiocarbamoyl)-L-cysteine (BNAC) as an internal standard, vortex-mixed for $3 \mathrm{~s}$, then combined with $50 \mu \mathrm{l}$ of icecold trifluoracetic acid and vortex-mixed again. The sample was centrifuged at $16000 \mathrm{~g}$ for $10 \mathrm{~min}$ at $4^{\circ} \mathrm{C}$ to pellet precipitated proteins. The supernatant was filtered with a $0 \cdot 2-\mu \mathrm{M}$ spin filter (Grace) at $10000 \mathrm{~g}$ for $5 \mathrm{~min}$ at $4^{\circ} \mathrm{C}$. A volume of $150 \mu \mathrm{l}$ of this filtered supernatant was transferred to a deactivated glass insert and autosampler vial (Agilent) for analysis. A 
volume of $0.5 \mathrm{ml}$ of ice-cold urine was combined with $25 \mu \mathrm{l}$ of $100 \mu \mathrm{M}$ BNAC, vortex-mixed for $3 \mathrm{~s}$ and centrifuged at $10000 \mathrm{~g}$ for $5 \mathrm{~min}$ at $4^{\circ} \mathrm{C}$. This filtered urine was mixed with $4.5 \mathrm{ml}$ of $10 \mathrm{~mm}$ ammonium acetate buffer at $4^{\circ} \mathrm{C}$ previously adjusted to pH 4.0 with acetic acid. A volume of $150 \mu$ of the sample was then transferred to a deactivated glass insert and autosampler vial for analysis.

Plasma and urine samples $(10 \mu \mathrm{l})$ were analysed by ultra high-performance liquid chromatography using an Agilent Zorbax SB-Aq column $(2.1 \times 100 \mathrm{~mm}, 1.8 \mu \mathrm{m})$ and an Agilent 6490 triple quadrupole mass spectrometer. Solvent A was $10 \mathrm{~mm}$ ammonium acetate adjusted to $\mathrm{pH} 4$ with acetic acid and solvent $\mathrm{B}$ was acetonitrile with $0 \cdot 1 \%$ acetic acid. The flow rate was $0.25 \mathrm{ml} / \mathrm{min}$ and the gradient began at $5 \% \mathrm{~B}$, increasing to $30 \%$ $\mathrm{B}$ over $5 \mathrm{~min}$ and returning to $5 \% \mathrm{~B}$ over an additional $6 \mathrm{~min}$. The UHPLC eluent was sprayed into the mass spectrometer source interface operating with electrospray ionisation in the positive mode. A total of nine metabolites were detected and measured by selected reaction monitoring collision-induced dissociation transitions: SF $(m / z \quad 178-114)$, sulforaphaneglutathione (SF-GSH, $m / z \quad 485-136)$, sulforaphanecysteineglycine (SF-CG, $m / z$ 356-136), sulforaphane-cysteine (SF-C, $m / z$ 299-136), sulforaphane- $N$-acetylcysteine (SF-NAC, $m / z$ 341-178), erucin-glutathione (ER-GSH, $m / z$ 469-179), erucin-cysteineglycine (ER-CG, $m / z$ 340-179), erucin-cysteine (ER-C, $m / z$ 283-103), erucin- $N$-acetylcysteine (ER-NAC, $m / z$ 325-164) and BNAC $(m / z 279-122)$. Source parameters were as follows: capillary voltage, $3000 \mathrm{~V}$; desolvation gas temperature and flow rate, $200^{\circ} \mathrm{C}$ and 14 litres $/ \mathrm{min}$, respectively; sheath gas temperature and flow rate, $250^{\circ} \mathrm{C}$ and 6 litres $/ \mathrm{min}$, respectively; and collision energy, 8-20 eV. External standard curves were produced using standards synthesised and purified by previously reported methods ${ }^{(33,34)}$ and used to quantify metabolites in plasma and urine. As was determined in a study using similar analytical techniques, ER could not be reproducibly measured and therefore was not included in the analysis $^{(24)}$

\section{Statistical analysis}

The plasma concentrations of SF and mercapturic acid pathway conjugates of SF and ER were adjusted for estimated plasma volumes ${ }^{(35,36)}$ and are presented as plasma mass of metabolites ( $\mu \mathrm{mol})$. We chose this approach so that differences in blood volume with body size would not skew the results. Rates of urinary excretion $(\mu \mathrm{mol} / \mathrm{h})$ of metabolites were determined by dividing the metabolite concentrations by the urine volumes from the time frames of collection. Plasma AUC was calculated using the linear trapezoidal method. The maximum plasma mass of metabolites $\left(M_{\max }\right)$ and time to reach this maximum $\left(T_{\max }\right)$ for each time point curve were determined by visual inspection. The elimination rate constant $(k)$ was estimated from nonlinear exponential decay curves fit to the elimination phase of plasma mass-time curves. ANOVA was performed using the GLIMMIX procedure in SAS (version 9.3; SAS Institute). For maximum plasma mass, L:M ratio and total plasma metabolites across time, whose observed values were continuously distributed across a fixed-interval (i.e. $[a, b]$ for any $a<b$ ) range, ANOVA models used a $\beta$ distribution with a logit function to link inferences to the original scale of the observed data values. For all other variables, observed values were continuously distributed like time values across a positive range $(0, \infty)$; ANOVA models used a $\gamma$ distribution with a log function to link inferences to the original scale of the observed data values. Plots of models' residuals were examined to verify goodness of fit. For models that analysed plasma and urinary metabolites with time, the following effects were included in the model: subject, period, sequence, hour, diet, BMI, GSTM1 genotype, sex, diet $\times$ hour, diet $\times$ BMI, diet $\times$ GSTM1 genotype, diet $\times$ sex, diet $\times$ BMI $\times$ hour, diet $\times$ GSTM1 genotype $\times$ hour and diet $\times \operatorname{sex} \times$ hour. All other models excluded the hour terms. Subject was modelled as a random effect and other effects were modelled as fixed effects. Pairwise $t$ tests $(P<0.05)$ were conducted at each time point to test for differences based on sex or GSTM1 genotype when the $\operatorname{diet} \times \operatorname{sex} \times$ hour or $\operatorname{diet} \times$ GSTM1 genotype $\times$ hour interaction was significant. For clear graphical depiction of the diet $\times$ BMI interaction, data were divided into two groups ( $n$ 9, for each) by median BMI $=26 \mathrm{~kg} / \mathrm{m}^{2}$. All significance tests of model effects and mean comparisons were conducted on the model's logit or log scale and reported on the original data scale as least squares means with $95 \%$ CI.

A diet $\times$ BMI interaction was observed for plasma and urinary metabolites, but the study was not powered to allow statistical detection of a diet $\times$ BMI interaction. A post hoc power analysis was conducted to identify a valid way to examine this interaction. On the basis of the philosophy that the eighteen original study observations can reasonably be assumed to be representative of observations that would have been measured if the original study had had thirty-six instead of eighteen subjects, all

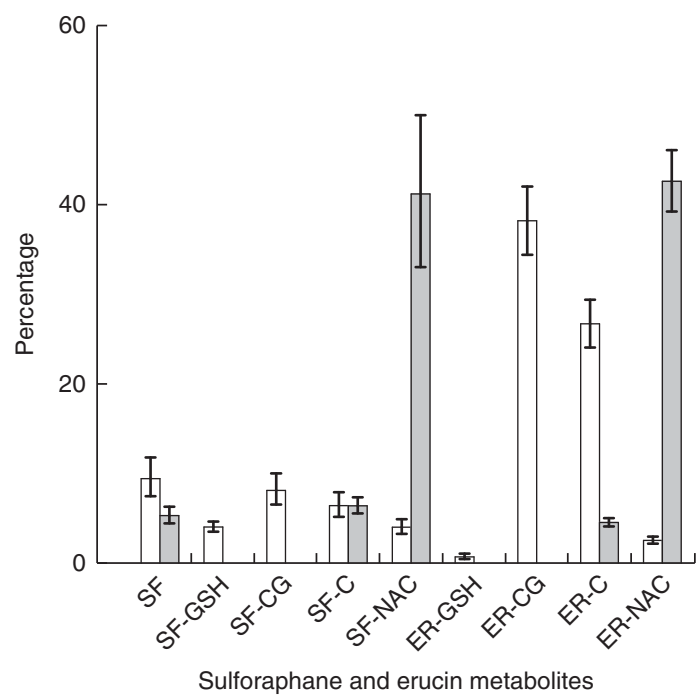

Fig. 3. Plasma AUC (over $24 \mathrm{~h}$ ) and urinary accumulation (over $24 \mathrm{~h}$ ) of metabolites of glucoraphanin and glucoerucin expressed as the percentage of total AUC and total urinary accumulation, respectively, of subjects who had consumed the diet with no broccoli. Values are least squares means and $95 \%$ Cl. SF, sulphoraphane; SF-GSH, sulphoraphane-glutathione; SF-CG, sulphoraphane-cysteineglycine; SF-C, sulphoraphane-cysteine; SF-NAC, sulphoraphane- $N$-acetylcysteine; ER-GSH, erucin-glutathione; ER-CG, erucin-cysteineglycine; ER, erucin-cysteine; ER-NAC, erucin- $N$ acetylcysteine. $\square$, Percentage plasma AUC; $\square$, percentage urinary accumulation. 
data analyses conducted for the original study (eighteen subjects) were repeated on the thirty-six observations (i.e. ' $x 2$ ' data) constructed by duplicating each of the original eighteen observations. False discovery rate (FDR $q$-values) were calculated for the $P$ values resulting from all tests on the ' $\chi$ ' data. All tests from the original study (eighteen subjects) with unadjusted $P$-value $<0.05$ also exhibited a $q$-value $<0.05$ in the ' $\chi$ ' post boc power analysis, indicating validity in reporting unadjusted $P$ values for all tests conducted for the original study.

\section{Results}

The quantities of individual metabolites of glucoraphanin and glucoerucin in plasma and urine, represented by percentage of total plasma AUC and percentage of total urinary accumulation, respectively, are presented in Fig. 3 for the NB diet. The main effect of diet was nonsignificant, and thus the results were similar for the DB diet (online Supplementary Fig. S3). Although glucoraphanin constituted $94 \%$ of the glucosinolates provided by glucoraphanin + glucoerucin in the broccoli challenge meal, SF and SF metabolites comprised 32 and $34 \%$ of plasma metabolites for the NB and DB diets, respectively, and made up 53 and $54 \%$ of urinary metabolites for the NB and DB diets, respectively. Metabolites measured in plasma were SF, SF-GSH, SF-CG, SF-C, SF-NAC, ER-GSH, ER-CG, ER-C and ER-NAC, of which ER-CG comprised $>37 \%$. In urine, SF-NAC and ER-NAC each comprised $>41 \%$ of metabolites, the remainder of which consisted of SF, SF-C, SF-CG and ER-C. SF-CG was $<0 \cdot 1 \%$ of urinary metabolites. Excreted urinary metabolites accumulated over $24 \mathrm{~h}$ accounted for $23 \%$ (NB diet) and $21 \%$ (DB diet) of the $103 \mu \mathrm{m}$ of parent glucosinolates (glucoraphanin and glucoerucin) provided in the broccoli challenge meal (Table 3).

Diet interacted with BMI such that for higher BMI, plasma AUC and $M_{\max }$ of total metabolites were higher for subjects on the NB diet compared with the DB diet, whereas for lower BMI plasma AUC and $M_{\max }$ were higher for subjects on the DB diet compared with those on the NB diet (Table 3; $P=0.0457$ for AUC; $P=0.0311$ for $\left.M_{\max } ; \mathrm{df}=15\right)$. To illustrate these relationships, mean AUC and mean $M_{\max }$ for subjects above and below median BMI $\left(26 \mathrm{~kg} / \mathrm{m}^{2}\right)$ are reported in Table 3. Pharmacokinetic data for individual metabolites are presented in online Supplementary Tables S1-S4. The interaction of diet with BMI was statistically significant $(P=0 \cdot 0464 ; \mathrm{df}=279)$ for the plasma mass of total metabolites over time (Fig. 4) and followed the plasma AUC and $M_{\max }$ relationships of higher values for subjects on the NB diet (compared with the DB diet) who had higher BMI and lower values for subjects on the DB diet (compared to NB diet) who had lower BMI. There was no effect of GSTM1 genotype or sex on any variables (data not shown).

The urinary excretion rates of the sum of SF and ER metabolites generally followed the same trends as levels of plasma metabolites. Although the main effect of diet was nonsignificant, the interaction of diet with BMI was significant at $P=0.0154$ (Fig. 5 ; $\mathrm{df}=108$ ). Urinary excretion rates for subjects with higher BMI were higher on the NB diet compared with the DB diet, and for lower BMI subjects, excretion rates were higher on the DB diet

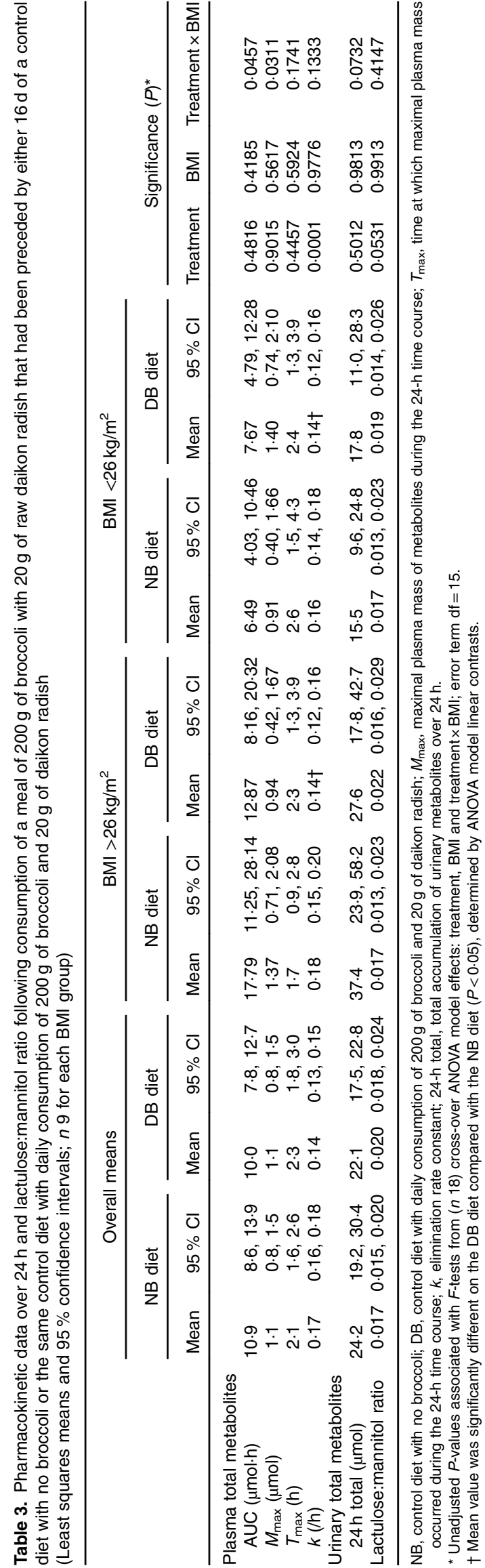


(a)

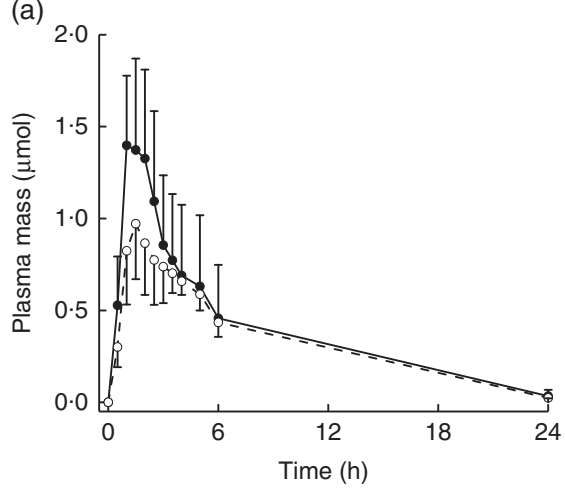

(b)

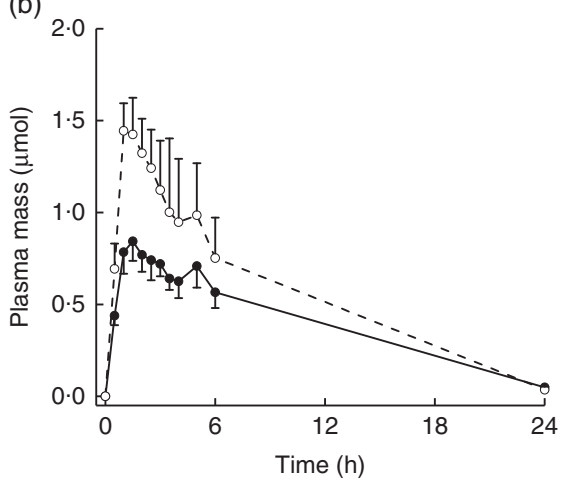

Fig. 4. Plasma mass (concentration/plasma volume) of total metabolites (sulphoraphane, sulphoraphane-glutathione, sulphoraphane-cysteineglycine, sulphoraphane-cysteine, sulphoraphane- $N$-acetylcysteine, erucin-glutathione, erucin-cysteineglycine, erucin-cysteine and erucin- $N$-acetylcysteine) with time for subjects with (a) BMI $<26 \mathrm{~kg} / \mathrm{m}^{2}$ and (b) BMI $>26 \mathrm{~kg} / \mathrm{m}^{2}$. Values are least squares means with upper $95 \%$ confidence limits shown above largest mean at a given time and lower $95 \%$ confidence limits shown below smallest mean at a given time. Treatment $\times$ BMI was statistically significant $(P=0.0464)$ as determined by ANOVA model linear contrasts. ——, Daily broccoli diet; $\_--,$, no broccoli diet.
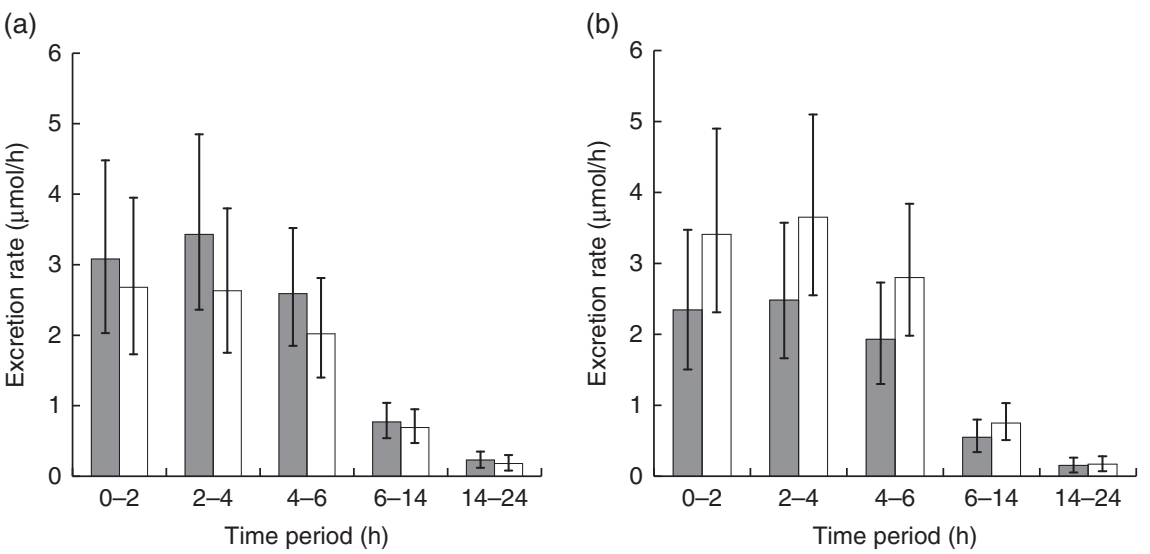

Fig. 5. Urinary excretion rate of total metabolites (sulphoraphane, sulphoraphane-glutathione, sulphoraphane-cysteineglycine, sulphoraphane-cysteine, sulphoraphane- $N$-acetylcysteine, erucin-glutathione, erucin-cysteineglycine, erucin-cysteine and erucin- $N$-acetylcysteine) during collection time periods for subjects with (a) BMI $<26 \mathrm{~kg} / \mathrm{m}^{2}$ and (b) BMl $>26 \mathrm{~kg} / \mathrm{m}^{2}$. Values are least squares means and $95 \% \mathrm{Cl}$. Treatment $\times \mathrm{BMI}$ was significant $(P=0.0154)$ as determined by ANOVA. $\square$, Daily broccoli diet; $\square$, no broccoli diet.

compared with the NB diet. The LM ratio was higher on the DB diet, and there was no relationship with BMI (Table 3).

\section{Discussion}

In this study, we investigated whether daily broccoli consumption modifies the metabolism of isothiocyanates and their metabolites as indicated by their levels in plasma and urine. We found that the effect of daily consumption depended on BMI. Following the broccoli challenge meal, the plasma AUC of total metabolites of low BMI subjects (BMI $<26 \mathrm{~kg} / \mathrm{m}^{2}$ ) was $17 \%$ higher when on the $\mathrm{DB}$ diet than when on the $\mathrm{NB}$ diet. In contrast, plasma AUC was $28 \%$ lower in high BMI subjects (BMI $>26 \mathrm{~kg} / \mathrm{m}^{2}$ ) on the DB diet compared with the NB diet.

This effect was also reflected by $M_{\max }$ of total metabolites, which was increased by $55 \%$ by the DB diet (compared with the NB diet) for low-BMI subjects but decreased by $33 \%$ by the DB diet for high-BMI subjects. $M_{\max }$ for $\mathrm{SF}+\mathrm{SF}$ metabolites followed similar trends (online Supplementary Table S2) and ranged from 0.56 (NB diet) to $0.91 \mu \mathrm{mol}$ (DB diet) for $\mathrm{BMI}$ $<26 \mathrm{~kg} / \mathrm{m}^{2}$ and from 0.66 (NB diet) to $0.42 \mu \mathrm{mol}$ (DB diet) for
BMI $>26 \mathrm{~kg} / \mathrm{m}^{2}$. In comparison, $M_{\max }$ for plasma SF + SF metabolites in a study providing $200 \mathrm{~g}$ of raw broccoli was $0.28 \mu \mathrm{mol}$ (calculated based on estimated plasma volume of 2.7 litres) ${ }^{(20)}$. This value is lower than our results and may be explained partly by differing total glucoraphanin amounts; we provided $98 \mu \mathrm{mol}$, whereas the cited study provided $61 \mu \mathrm{mol}$.

The optimal levels of plasma SF and SF metabolites for conferring health benefits have not been established but likely would depend on the tissue and possible benefit in question. However, pre-clinical studies suggest that the effects of SF and SF metabolites are dose dependent ${ }^{(37,38)}$. Thus, the interactive effect of daily broccoli consumption with BMI on plasma levels of SF and SF metabolites may have a corresponding influence on molecular mechanisms related to disease risk.

Evidence is accumulating that repeated intake of some foods may alter the absorption and metabolism of potentially healthpromoting phytonutrients. A clinical study showed that daily administration of $800 \mathrm{mg}$ of epigallocatechin gallate for 4 weeks resulted in $60 \%$ greater plasma AUC at the end compared with the start of the study ${ }^{(39)}$. Another clinical study demonstrated not only an effect of repeated intake on flavonoid absorption, 
but also an effect of BMI. In that study, lean volunteers had a greater plasma flavonoid response after consuming mixed grape polyphenols for $10 \mathrm{~d}$ compared with plasma response to a dose given before the $10 \mathrm{~d}$ of repeated exposure, whereas obese volunteers showed no adaptation ${ }^{(29)}$. Much less is known about how repeated intake of glucosinolate-rich foods affects absorption and metabolism. In one study, $300 \mathrm{ml}$ of liquefied broccoli florets was provided to six volunteers daily for $10 \mathrm{~d}$, and the pharmacokinetic response of plasma SF did not differ between days 1 and $10^{(40)}$. Notably, this study did not investigate the relationship of plasma response with BMI.

SF originates from the catabolism of glucoraphanin mediated by plant or gut-bacterial myrosinase and perhaps from the oxidation of ER originating from glucoerucin. SF and ER are metabolised through the mercapturic acid pathway in enterocytes and the liver, beginning with the GST-mediated conjugation of the isothiocyanate with $\mathrm{GSH}^{(41)}$. The diet effect we observed in response to BMI may have been driven by mechanisms that altered the fraction of glucosinolates that were converted to isothiocyanates. We considered that prior exposure to SF might up-regulate GST in the enterocyte, thereby enhancing metabolism and secretion back into the gut as has been observed in vivo ${ }^{(42)}$, although the relationship of this mechanism to BMI has not been established. Because the gut microbiome varies with $\mathrm{BMI}^{(43,44)}$, it is possible that the interactions of diet with BMI for isothiocyanate metabolite levels reflect interactions in the intestinal microbiota. SF is presumed to be the first metabolite that is generated from glucoraphanin following broccoli intake and is the parent of the thiol conjugates. Therefore, microbial changes altering the generation of $\mathrm{SF}$ would be reflected in the sum of all metabolites. For example, changes in gut microbiota with consumption of Brassica vegetables have been reported ${ }^{(45)}$, and changes in myrosinaseexpressing bacteria with daily broccoli consumption could increase the quantity of isothiocyanates available for absorption $^{(46)}$. However, the peak plasma levels of most metabolites were from approximately 1 to $3 \mathrm{~h}$, which is consistent with absorption before the broccoli bolus reaching the colon (Table 3). In contrast, the time of maximum SF levels occurred at approximately $5 \mathrm{~h}$ for both NB and DB groups (online Supplementary Table S3), which may be the result of absorption farther down the gastrointestinal tract, either owing to earlier excretion of the GSH complex or to newly metabolised glucoraphanin by the microbiome, leading to release of SF for reabsorption. It is possible that appearance of isothiocyanates in plasma at the later time points was influenced by changes in the capacity of microbiota to hydrolyse glucoraphanin and glucoerucin in the present study. Because the gut microbiome varies with $\mathrm{BMI}^{(43,44)}$, it is possible that the interaction of diet with BMI for isothiocyanate metabolite levels reflects similar interactions in the intestinal microbiota.

Changes in gut transit time induced by daily broccoli feeding may also be a factor causing the observed changes in plasma metabolite levels and urinary excretion of SF and other metabolites. Mean gut transit times are negatively correlated with BMI ${ }^{(47)}$. Dietary fibre decreases transit time ${ }^{(48)}$ and dietary fibre and transit time independently modify microbiota composition ${ }^{(49-51)}$. Thus, the $16 \mathrm{~d}$ of daily broccoli consumption with the additional $5 \mathrm{~g} / \mathrm{d}$ of dietary fibre (Table 2) above that in the NB diet may have altered gut microbiota such that transit times differed compared with the NB diet. When microbiota were transplanted from healthy humans and individuals with chronic constipation to pseudo-germ-free mice, mice treated with microbiota from slow-transit constipation donors had delayed gastrointestinal transit time compared with healthy controls ${ }^{(52)}$. Thus, both the added fibre and bioactive glucosinolate-derived metabolites ${ }^{(46)}$ of the DB diet may have modified microbial composition and in turn the gut transit time during which metabolites could be absorbed. Interestingly, genistein was more highly absorbed following a single dose of soya milk powder in women with relatively rapid gut transit times ${ }^{(53)}$, suggesting that at least for the case of genistein, absorption is related to the gut lumenal environment and not simply to the rate of passage.

BMI reflects differences in absorption, distribution and metabolism of a number of dietary components. Fat-soluble nutrients generally are retained in adipose tissue and larger adipose depots may result in reduced elimination rates. $\beta$-Carotene and $\alpha$-tocopherol absorption in healthy overweight subjects consuming extracts of mixed fruits and vegetables and the rates of depletion showed an inverse correlation with general adiposity ${ }^{(54)}$. In healthy, post-menopausal women, a randomised cross-over study showed that BMI, percentage of body fat and absolute amounts of central and peripheral fat were inversely related to serum folate $^{(55)}$. Vitamin K levels were positively correlated with adipose tissue and inversely correlated with circulating markers of vitamin $\mathrm{K}$ status $^{(56)}$. Because not all of the glucosinolate dose provided was recovered in 24-h urine, it is possible that some of the SF was retained in adipose tissue, although the water-soluble thiol conjugates likely would not be so retained. However, such a phenomenon does not explain why repeated intake of broccoli changes the plasma pharmacokinetic and urinary excretion responses depending on BMI level, as subjects were weight-stable throughout the study. Greater adiposity has also been associated with greater gut permeability ${ }^{(57)}$, which conceivably could alter plasma and urinary isothiocyanate metabolites. However, the LM ratio, which reflects gut permeability, was not related with BMI and thus did not provide evidence for this explanation.

We note several limitations to this study. One limitation is that because we did not anticipate that treatment would interact with BMI we did not conduct measurements of body fat percentage by dual-energy X-ray absorptiometry and therefore could not determine whether the contribution of BMI was more precisely a result of body fat. A second limitation is that the fibre content of the NB diet was not matched to that of the DB diet, and the difference in fibre may have had a role in the absorption of glucosinolate metabolites. A third limitation is that we did not use fresh broccoli in this study, as might be more generally applicable to dietary eating patterns. To ensure homogeneity of the broccoli throughout the study, we procured a single batch of frozen broccoli and used daikon radish as the myrosinase source. Subjects consumed slightly more food on the DB diet compared with the NB diet. The average energy content in a pound of body weight is approximately $14644 \mathrm{~kJ}$. The added energy from the $200 \mathrm{~g}$ of broccoli and $20 \mathrm{~g}$ of daikon radish totalled $301 \mathrm{~kJ} / \mathrm{d}$. Over $16 \mathrm{~d}$ of feeding before the broccoli pharmacokinetic challenge meal, $301 \mathrm{~kJ} / \mathrm{d}$ is equivalent to $4820 \mathrm{~kJ}$ over the study period, which is about one-third the 
energy in a pound of body weight. The average human weighs around $80 \mathrm{~kg}$ (approximately $176 \mathrm{lbs}$ ); $1 / 3 \mathrm{lb}$ is only $0.2 \%$ of $176 \mathrm{lbs}$ and thus is insignificant compared with $176 \mathrm{lbs}$. We believe it is highly unlikely that this affected the results. Finally, we report $P$ values unadjusted for multiplicity, based on post hoc power analyses indicating that FDR $q$-values from duplicate (thirty-six subjects) analyses were consistent with unadjusted $P$ value results from the original eighteen subjects.

In conclusion, plasma and urinary levels of SF and mercapturic acid pathway products of SF and ER following a broccoli challenge meal were altered when preceded by $16 \mathrm{~d}$ of daily broccoli ingestion, and the effect depended on BMI. These results point towards the need for, and the challenge of, developing individualised dietary recommendations for consumption of broccoli and perhaps other Brassica vegetables. Where adaptation to daily broccoli consumption resulted in decreased plasma and urinary response (e.g. those with higher BMI), more research is needed to determine whether regular broccoli consumption resulting in lower levels of isothiocyanate metabolites after each meal is more beneficial than infrequent consumption resulting in higher levels after an occasional broccoli meal.

\section{Acknowledgements}

The authors thank Dawn J. Harrison and Meredith A. Capuco for conducting the analytical analyses.

This work was supported by the United States Department of Agriculture (USDA) (J. A. N., 8040-51000-056-00D) and the National Cancer Institute (ACN16003).

The authors' contributions are as follows: C. S. C., J. A. N., E. H. J., H. E. S. and S. A. R. designed the research; C. S. C. and J. A. N. conducted the research; B. T. V. performed the statistical analysis; C. S. C. wrote the paper and had primary responsibility for the final content. All authors approved the final version of the manuscript.

The authors declare that there are no conflicts of interest.

The mention of trade names or commercial products in this publication is solely for the purpose of providing specific information and does not imply recommendation or endorsement by the USDA. The USDA is an equal opportunity provider and employer.

\section{Supplementary material}

For supplementary material/s referred to in this article, please visit https://doi.org/10.1017/S0007114518002921

\section{References}

1. Chen YJ, Wallig MA \& Jeffery EH (2016) Dietary broccoli lessens development of fatty liver and liver cancer in mice given diethylnitrosamine and fed a western or control diet. J Nutr 146, 542-550.

2. Tse G \& Eslick GD (2014) Cruciferous vegetables and risk of colorectal neoplasms: a systematic review and meta-analysis. Nutr Cancer 66, 128-139.

3. Wu QJ, Yang Y, Vogtmann E, et al. (2013) Cruciferous vegetables intake and the risk of colorectal cancer: a meta-analysis of observational studies. Ann Oncol 24, 1079-1087.
4. Liu X \& Lv K (2013) Cruciferous vegetables intake is inversely associated with risk of breast cancer: a meta-analysis. Breast 22, 309-313.

5. Terry P, Vainio H, Wolk A, et al. (2002) Dietary factors in relation to endometrial cancer: a nationwide case-control study in Sweden. Nutr Cancer 42, 25-32.

6. Han B, Li X \& Yu T (2014) Cruciferous vegetables consumption and the risk of ovarian cancer: a meta-analysis of observational studies. Diagn Pathol 9, 7.

7. Carpenter CL, Yu MC \& London SJ (2009) Dietary isothiocyanates, glutathione $S$-transferase M1 (GSTM1), and lung cancer risk in African Americans and Caucasians from Los Angeles County, California. Nutr Cancer 61, 492-499.

8. Lam TK, Ruczinski I, Helzlsouer KJ, et al. (2010) Cruciferous vegetable intake and lung cancer risk: a nested case-control study matched on cigarette smoking. Cancer Epidemiol Biomarkers Prev 19, 2534-2540.

9. Thimmulappa RK, Mai KH, Srisuma S, et al. (2002) Identification of Nrf2-regulated genes induced by the chemopreventive agent sulforaphane by oligonucleotide microarray. Cancer Res 62, 5196-5203.

10. Fahey JW, Wehage SL, Holtzclaw WD, et al. (2012) Protection of humans by plant glucosinolates: efficiency of conversion of glucosinolates to isothiocyanates by the gastrointestinal microflora. Cancer Prev Res 5, 603-611.

11. Sakao K \& Singh SV (2012) D,L-Sulforaphane-induced apoptosis in human breast cancer cells is regulated by the adapter protein p66sup Shc. J Cell Biochem 113, 599-610.

12. Nian H, Delage B, Ho E, et al. (2009) Modulation of histone deacetylase activity by dietary isothiocyanates and allyl sulfides: studies with sulforaphane and garlic organosulfur compounds. Environ Mol Mutagen 50, 213-221.

13. Greaney AJ, Maier NK, Leppla SH, et al. (2016) Sulforaphane inhibits multiple inflammasomes through an Nrf2independent mechanism. J Leukoc Biol 99, 189-199.

14. Zhang Y, Talalay P, Cho C, et al. (1992) A major inducer of anticarcinogenic protective enzymes from broccoli: isolation and elucidation of structure. Proc Natl Acad Sci U S A 89, 2399-2403.

15. Gamet-Payrastre L, Li P, Lumeau S, et al. (2000) Sulforaphane, a naturally occurring isothiocyanate, induces cell cycle arrest and apoptosis in HT29 human colon cancer cells. Cancer Res 60, 1426-1433.

16. Gasper AV, Traka M, Bacon JR, et al. (2007) Consuming broccoli does not induce genes associated with xenobiotic metabolism and cell cycle control in human gastric mucosa. J Nutr 137, 1718-1724.

17. Gasper AV, Al-Janobi A, Smith JA, et al. (2005) Glutathione $S$-transferase M1 polymorphism and metabolism of sulforaphane from standard and high-glucosinolate broccoli. Am J Clin Nutr 82, 1283-1291.

18. Seow A, Shi CY, Chung FL, et al. (1998) Urinary total isothiocyanate (ITC) in a population-based sample of middleaged and older Chinese in Singapore: relationship with dietary total ITC and glutathione $S$-transferase M1/T1/P1 genotypes. Cancer Epidemiol Biomarkers Prev 7, 775-781.

19. Faulkner K, Mithen R \& Williamson G (1998) Selective increase of the potential anticarcinogen 4-methylsulphinylbutyl glucosinolate in broccoli. Carcinogenesis 19, 605-609.

20. Vermeulen M, Klöpping-Ketelaars IWAA, van den Berg R, et al. (2008) Bioavailability and kinetics of sulforaphane in humans after consumption of cooked versus raw broccoli. J Agric Food Chem 56, 10505-10509.

21. Oliviero $\mathrm{T}$, Verkerk $\mathrm{R}$, Vermeulen $\mathrm{M}$, et al. (2014) In vivo formation and bioavailability of isothiocyanates from glucosinolates in broccoli as affected by processing conditions. Mol Nutr Food Res 58, 1447-1456. 
22. Li F, Hullar MAJ, Beresford SAA, et al. (2011) Variation of glucoraphanin metabolism in vivo and ex vivo by human gut bacteria. Br J Nutr 106, 408-416.

23. Angelino D, Dosz EB, Sun J, et al. (2015) Myrosinasedependent and -independent formation and control of isothiocyanate products of glucosinolate hydrolysis. Front Plant Sci 6, 1-6.

24. Clarke JD, Hsu A, Riedl K, et al. (2011) Bioavailability and inter-conversion of sulforaphane and erucin in human subjects consuming broccoli sprouts or broccoli supplement in a cross-over study design. Pharmacol Res 64, 456-463.

25. Platz S, Piberger AL, Budnowski J, et al. (2015) Bioavailability and biotransformation of sulforaphane and erucin metabolites in different biological matrices determined by LC-MS-MS. Anal Bioanal Chem 407, 1819-1829.

26. Dosz EB \& Jeffery EH (2013) Commercially produced frozen broccoli lacks the ability to form sulforaphane. J Funct Foods 5, 987-990

27. Saha S, Hollands W, Teucher B, et al. (2012) Isothiocyanate concentrations and interconversion of sulforaphane to erucin in human subjects after consumption of commercial frozen broccoli compared to fresh broccoli. Mol Nutr Food Res 56 , 1906-1916.

28. Prosky L, Asp NG, Furda I, et al. (1985) Determination of total dietary fiber in food and food products: collaborative study. $J$ Assoc Off Anal Chem 68, 677-679.

29. Novotny JA, Chen TY, Terekhov AI, et al. (2017) The effect of obesity and repeated exposure on pharmacokinetic response to grape polyphenols in humans. Mol Nutr Food Res 61, 1700043.

30. Charron CS, Clevidence BA, Albaugh GA, et al. (2013) Assessment of DNA damage and repair in adults consuming allyl isothiocyanate or Brassica vegetables. I Nutr Biochem 24, 894-902.

31. Miki K, Butler R, Moore D, et al. (1996) Rapid and simultaneous quantification of rhamnose, mannitol, and lactulose in urine by HPLC for estimating intestinal permeability in pediatric practice. Clin Chem 42, 71-75.

32. Janobi AAA, Mithen RF, Gasper AV, et al. (2006) Quantitative measurement of sulforaphane, iberin and their mercapturic acid pathway metabolites in human plasma and urine using liquid chromatography-tandem electrospray ionisation mass spectrometry. J Chromatogr B 844, 223-234.

33. Kassahun K, Davis M, Hu P, et al. (1997) Biotransformation of the naturally occurring isothiocyanate sulforaphane in the rat: identification of phase I metabolites and glutathione conjugates. Chem Res Toxicol 10, 1228-1233.

34. Egner PA, Kensler TW, Chen J-G, et al. (2008) Quantification of sulforaphane mercapturic acid pathway conjugates in human urine by high-performance liquid chromatography and isotope-dilution tandem mass spectrometry. Chem Res Toxicol 21, 1991-1996.

35. Lemmens HJM, Bernstein DP \& Brodsky JB (2006) Estimating blood volume in obese and morbidly obese patients. Obes Surg 16, 773-776.

36. Dill DB \& Costill DL (1974) Calculation of percentage changes in volumes of blood, plasma, and red cells in dehydration. J Appl Physiol 37, 247-248.

37. Juge N, Mithen R \& Traka M (2007) Molecular basis for chemoprevention by sulforaphane: a comprehensive review. Cell Mol Life Sci 64, 1105-1127.

38. Wu S, Zhou Y, Yang G, et al. (2017) Sulforaphane-cysteine induces apoptosis by sustained activation of ERK1/2 and caspase 3 in human glioblastoma U373MG and U87MG cells. Oncol Rep 37, 2829-2838.

39. Chow HHS, Cai Y, Hakim IA, et al. (2003) Pharmacokinetics and safety of green tea polyphenols after multiple-dose administration of epigallocatechin gallate and polyphenon $\mathrm{E}$ in healthy individuals. Clin Cancer Res 9, 3312-3319.

40. Hanlon N, Coldham N, Gielbert A, et al. (2009) Repeated intake of broccoli does not lead to higher plasma levels of sulforaphane in human volunteers. Cancer Lett 284, 15-20.

41. Baenas N, Silván JM, Medina S, et al. (2015) Metabolism and antiproliferative effects of sulforaphane and broccoli sprouts in human intestinal (Caco-2) and hepatic (HepG2) cells. Phytochem Rev 14, 1035-1044.

42. Petri N, Tannergren C, Holst B, et al. (2003) Absorption/ metabolism of sulforaphane and quercetin, and regulation of phase II enzymes, in human jejunum in vivo. Drug Metab Dispos 31, 805-813.

43. Dominianni C, Sinha R, Goedert JJ, et al. (2015) Sex, body mass index, and dietary fiber intake influence the human gut microbiome. PLOS ONE 10, e0124599.

44. Haro C, Rangel-Zúñiga OA, Alcalá-Díaz JF, et al. (2016) Intestinal microbiota is influenced by gender and body mass index. PLOS ONE 11, e0154090.

45. Li F, Hullar MAJ, Schwarz Y, et al. (2009) Human gut bacterial communities are altered by addition of cruciferous vegetables to a controlled fruit- and vegetable-free diet. J Nutr 139, $1685-1691$

46. Liu X, Wang Y, Hoeflinger JL, et al. (2017) Dietary broccoli alters rat cecal microbiota to improve glucoraphanin hydrolysis to bioactive isothiocyanates. Nutrients 9, 262-274.

47. Probert CSJ, Emmett PM \& Heaton KW (1995) Some determinants of whole-gut transit time: a population-based study. OJM 88, 311-315.

48. Anderson JW, Baird P, Davis RH Jr, et al. (2009) Health benefits of dietary fiber. Nutr Rev 67, 188-205.

49. Chiang EPI, Chiu SC, Pai MH, et al. (2013) Organosulfur garlic compounds induce neovasculogenesis in human endothelial progenitor cells through a modulation of microRNA 221 and the PI3-K/Akt signaling pathways. J Agric Food Chem 61, 4839-4849.

50. Tottey W, Feria-Gervasio D, Gaci N, et al. (2017) Colonic transit time is a driven force of the gut microbiota composition and metabolism: in vitro evidence. J Neurogastroenterol Motil 23, 124-134

51. Stephen AM, Wiggins HS \& Cummings JH (1987) Effect of changing transit time on colonic microbial metabolism in man. Gut 28, 601-609.

52. Ge X, Zhao W, Ding C, et al. (2017) Potential role of fecal microbiota from patients with slow transit constipation in the regulation of gastrointestinal motility. Sci Rep 7, 441.

53. Zheng Y, Hu J, Murphy PA, et al. (2003) Rapid gut transit time and slow fecal isoflavone disappearance phenotype are associated with greater genistein bioavailability in women. J Nutr 133, 3110-3116.

54. Wise JA, Kaats GR, Preuss HG, et al. (2009) $\beta$-Carotene and $\alpha$-tocopherol in healthy overweight adults; depletion kinetics are correlated with adiposity. Int J Food Sci Nutr 60, 65-75.

55. Mahabir S, Ettinger S, Johnson L, et al. (2008) Measures of adiposity and body fat distribution in relation to serum folate levels in postmenopausal women in a feeding study. Eur J Clin Nutr 62, 644-650.

56. Shea MK, Booth SL, Gundberg CM, et al. (2010) Adulthood obesity is positively associated with adipose tissue concentrations of vitamin $\mathrm{K}$ and inversely associated with circulating indicators of vitamin $\mathrm{K}$ status in men and women. J Nutr 140, 1029-1034.

57. Yang B, Bostick RM, Tran HQ, et al. (2016) Circulating biomarkers of gut barrier function: correlates and nonresponse to calcium supplementation among colon adenoma patients. Cancer Epidemiol Biomarkers Prev 25, 318-326. 